\title{
Modified Newton's method using harmonic mean for solving nonlinear equations
}

\author{
J. Jayakumarand Kalyanasundaram M. \\ Department of Mathematics, Pondicherry Engineering College, Pondicherry 605 014, India.
}

\begin{abstract}
Different modifications in the Newton's method with cubic convergence have become popular iterative methods to find the roots of non-linear equations. In this paper, a modified Newton's method for solving a single nonlinear equation is proposed. This method uses harmonic mean while using Simpson's integration rule, thus replacing $f^{\prime}(x)$ in the classical Newton's method. The convergence of the proposed method is found to be order three. Numerical examples are provided to compare e the efficiency of the method with few other cubic convergent methods.
\end{abstract}

Keywords: Non-linear equation, Iterative Methods, Newton's Method, Third order convergence.

\section{Introduction} equation

A frequently occurringand most important problem of applied mathematicsis to find a root of the

$$
f(x)=0
$$

wheref $: \mathrm{I} \subseteq \mathrm{R} \rightarrow \mathrm{R}$ for an open interval $\mathrm{I}$ is a scalar function.

Newton's method is one of the famous iterative methods to solve equation (1). It is well known that it has quadratic convergence. The iterative formula of Newton's method is given by

$$
x_{n+1}^{*}=x_{n}-\frac{f\left(x_{n}\right)}{f^{\prime}\left(x_{n}\right)}, n=0,1,2 \ldots
$$

By improving Newton's method, Chebyshev [1] derived a cubic convergent iterative method which requires computing of second derivative of the function (1). A prolific advancement in this area has happened in the last decade where lots of modifications in Newton's method are proposed without using second derivative. First among this is arithmetic mean Newton's method [2] derived using the trapezoid rule. Hasanov et al [3] modified Newton's method with a third order convergent method by using Simpson's rule. Nedzhibov [4] gave several classes of iterative methods using different quadrature rules. Frontini et al [5] extended the results of [2] and got methods of order three independent of the integration formula used. Ozban [6] derived some new variants of Newton's method by using Harmonic mean instead of arithmetic mean and the midpoint integration instead of the trapezoidal formula. Using Newton's theorem for the inverse function, Homeier [7] derived a new class of Newton-type methods. Babajee et al [8] listed eight different variants of Newton's method and the relationship between them. They analyzed the properties of arithmetic mean Newton's method and explain the third order accuracy via the Tailor's expansion. Kou Jisheng [9] gave a new variant of Newton's method from the Harmonic mean and midpoint Newton's method [6]. Tibor et al [10] suggested geometric mean Newton's method for simple and multiple roots and showed this method is of order three for simple roots and order one for multiple roots. Ababneh [11] proposed a modified Newton's method based on contra harmonic mean. The advantage of all the above methods is that they use only the first derivative of the function and has cubic convergence.

In this paper,we propose amodification in the method of[3] including Harmonic mean instead of Arithmetic meanwhile using the Simpson's 1/3 rule of integration for the equation (3). The proposed new method has the advantage of evaluating only the firstderivativeand less number of iterations to achieve third order accuracy.In Section II, we present some definitions related to our study. In Section III, some known variants of Newton's method are discussed. Section IV presents the new method and its analysis of convergence. Finally, Section V gives numerical results and a discussion is carried out in section VI.

\section{Definitions}

if

Definition 2.1 [2]: Let $\alpha \in R, x_{n} \in R, \mathrm{n}=0,1,2, \ldots$. Then the sequence $\left\{x_{n}\right\}$ is said to converge to $\alpha$

$$
\lim _{n \rightarrow \infty}\left|x_{n}-\alpha\right|=0
$$

If, in addition, there exist a constantc $\geq 0$, an integer $n_{0} \geq 0$ and $\mathrm{p} \geq 0$ such that for all $n>0$,

$$
\left|x_{n+1}-\alpha\right| \leq c\left|x_{n}-\alpha\right|^{\mathrm{P}}
$$


then $\left\{x_{n}\right\}$ is said to converge to $\alpha$ with order at least $\mathrm{p}$. If $\mathrm{p}=2$ or 3 , the convergence is said to be quadratic or cubic respectively.

Definition 2.2 [2]: Let $\alpha$ be a root of the function (1)and suppose that $x_{n-1}, x_{n}$ and $x_{n+1}$ are three successive iterations closer to the root $\alpha$. Then, the computational order of convergence (COC) $\rho$ can be approximated using the formula $\rho \approx \frac{\ln \left|\left(x_{n+1}-\alpha\right) /\left(x_{n}-\alpha\right)\right|}{\ln \left|\left(x_{n}-\alpha\right) /\left(x_{n-1}-\alpha\right)\right|}$.

\section{Known Variants of Newton's Method}

Let $\alpha$ be a simple root of a sufficiently differentiable function $f(x)$. Consider the numerical solution of the function $f(x)=0$. From the Newton's theorem, we have

$f(x)=f\left(x_{n}\right)+\int_{x_{n}}^{x} f^{\prime}(t) d t$.

Arithmetic Mean Newton's method (AN): By using the trapezoidal rule in (3), we obtain

$\int_{x_{n}}^{x} f^{\prime}(t) d t \approx \frac{\left(x-x_{n}\right)}{2 m}\left[f^{\prime}\left(x_{n}\right)+2 \sum_{i=1}^{m-1} f^{\prime}\left(x_{n}-\frac{i f\left(x_{n}\right)}{m f^{\prime}\left(x_{n}\right)}\right)+f^{\prime}(x)\right]$.

From equations (3) and (4), a new approximation $x_{\mathrm{n}+1}$ for $x$ is obtained and for $\mathrm{m}=1$, we get

$$
x_{n+1}=x_{n}-\frac{2 f\left(x_{n}\right)}{f^{\prime}\left(x_{n}\right)+f^{\prime}\left(x_{n+1}^{*}\right)}, n=0,1,2 \ldots
$$

Equation (5) is the arithmetic mean Newton's method [2]. Since equation (5) is implicit, we can overcome this implicit nature by the use of Newton's iterative step (2).

Harmonic Mean Newton's method (HN): In equation (5), if we use the harmonic mean instead of arithmetic mean, we obtain harmonic mean Newton's method [6]

$$
x_{n+1}=x_{n}-\frac{f\left(x_{n}\right)\left(f^{\prime}\left(x_{n}\right)+f^{\prime}\left(x_{n+1}^{*}\right)\right)}{2 f^{\prime}\left(x_{n}\right) f^{\prime}\left(x_{n+1}^{*}\right)}, n=0,1,2 \ldots
$$

where $x_{n+1}^{*}$ is calculated from (2).

Midpoint Newton's method (MN):If the integral in (3) is approximated using the midpoint integration rule instead of trapezoidal rule, we get the midpoint Newton's method [6]

$$
x_{n+1}=x_{n}-\frac{f\left(x_{n}\right)}{f^{\prime}\left(\frac{x_{n}+x_{n+1}^{*}}{2}\right)}, \mathrm{n}=0,1,2, \ldots
$$

wherex $\mathrm{n}_{\mathrm{n}+1}^{*}$ is calculated from (2).

Trapezoidal-Newton's Method (TN): Simplifying and computing $x_{n+1}$ from equations (3) and (4), one obtains a new approximation for $\mathrm{m}=2$ as

$$
x_{n+1}=x_{n}-\frac{4 f\left(x_{n}\right)}{f^{\prime}\left(x_{n}\right)+2 f^{\prime}\left(\frac{x_{n+1}^{*}+x_{n}}{2}\right)+f^{\prime}\left(x_{n+1}^{*}\right)}, n=0,1,2 \ldots .
$$

where $x_{n+1}^{*}$ is calculated from (2). The method (7) is called Trapezoidal-Newton's method [4].

Simpson-Newton's Method (SN): The Simpson's $\frac{1}{3}$ integration rule given by

$$
\int_{x_{n}}^{x} f^{\prime}(t) d t \approx \frac{\left(x-x_{n}\right)}{6}\left[f^{\prime}\left(x_{n}\right)+4 f^{\prime}\left(\frac{x+x_{n}}{2}\right)+f^{\prime}(x)\right],
$$

is used to find the value of the integral in (3), then we get Simpson-Newton's method [3].Simplifying and computing for $x_{\mathrm{n}+1}$ from equation (8), one obtains a new approximation

$$
x_{n+1}=x_{n}-\frac{6 f\left(x_{n}\right)}{f^{\prime}\left(x_{n}\right)+4 f^{\prime}\left(\frac{x_{n}+1+x_{n}}{2}\right)+f^{\prime}\left(x_{n+1}\right)}, n=0,1,2 \ldots .
$$

Since equation (9) is implicit, replacing $x_{n+1}$ with $x_{n+1}^{*}$ (calculated from (2)), we get

$$
x_{n+1}=x_{n}-\frac{6 f\left(x_{n}\right)}{f^{\prime}\left(x_{n+1}^{*}\right)+4 f^{\prime}\left(\frac{x_{n+1}^{*}+x_{n}}{2}\right)+f^{\prime}\left(x_{n}\right)}, n=0,1,2 \ldots
$$

\section{Modified Newton's Method and its analysis ofconvergence}

We obtain the present method as follows: Rewrite equation (10) as

$$
x_{n+1}=x_{n}-\frac{3 f\left(x_{n}\right)}{\left[\frac{f^{\prime}\left(x_{n}\right)+f^{\prime}\left(x_{n+1}^{*}\right)}{2}\right]+2 f^{\prime}\left(\frac{x_{n+1}^{*}+x_{n}}{2}\right)}
$$


Wereplace harmonic mean [6] instead of arithmetic mean in the first part of the denominator. Then we obtain a modified Newton's method calledHarmonic-Simpson-Newton's method (HSN)

$$
x_{n+1}=x_{n}-\frac{3 f\left(x_{n}\right)}{\left[\frac{2 f^{\prime}\left(x_{n}\right) f^{\prime}\left(x_{n+1}^{*}\right)}{f^{\prime}\left(x_{n}\right)+f^{\prime}\left(x_{n+1}^{*}\right)}\right]+2 f^{\prime}\left(\frac{x_{n+1}^{*}+x_{n}}{2}\right)}
$$

where $x_{n+1}^{*}$ is calculated from (2).

In the following theorem, we prove the cubic convergence of the present method(11).

Theorem 4.1:Let $\alpha \in I$, be a simple zero of a sufficiently differentiablefunction $f: I \subseteq R \rightarrow R$ for an open intervalI. If $x_{0}$ is sufficiently nearerto $\alpha$, then the method (11) has third order convergence.

Proof.Let $\alpha$ be a simple zero of function $f(x)=0$. (That is, $f(\alpha)=0$ and $f^{\prime}(\alpha) \neq 0$ ).

By expanding $f\left(x_{n}\right)$ and $f^{\prime}\left(x_{n}\right)$ by Taylor seriesabout $\alpha$, we obtain

$f\left(x_{n}\right)=f^{\prime}(\alpha)\left[e_{n}+c_{2} e_{n}^{2}+c_{3} e_{3}^{3}+O\left(e_{n}^{4}\right) \ldots\right]$

$f^{\prime}\left(x_{n}\right)=f^{\prime}(\alpha)\left[1+2 c_{2} e_{n}+3 c_{3} e_{n}^{2}+4 c_{4} e_{n}^{3}+O\left(e_{n}^{4}\right) \ldots\right]$

where $c_{k}=\frac{f^{(k)}(\alpha)}{k ! f^{\prime}(\alpha)}, k=2,3, \ldots$ and $e_{n}=x_{n}-\alpha$.

Dividing equation (12) and (13), we get

$$
\begin{aligned}
& \frac{f\left(x_{n}\right)}{f^{\prime}\left(x_{n}\right)}=e_{n}-c_{2} e_{n}^{2}+2\left(c_{2}^{2}-c_{3}\right) e_{n}^{3}+O\left(e_{n}^{4}\right) . \text { Hencefrom (2), we get } \\
& x_{n+1}^{*}=\alpha+c_{2} e_{n}^{2}+2\left(c_{3}-c_{2}^{2}\right) e_{n}^{3}+O\left(e_{n}^{4}\right)
\end{aligned}
$$

Again expanding $f^{\prime}\left(x_{n+1}^{*}\right)$ about $\alpha$ and using (14),we get

$f^{\prime}\left(x_{n+1}^{*}\right)=f^{\prime}(\alpha)\left[1+2 \mathrm{c}_{2}^{2} \mathrm{e}_{\mathrm{n}}^{2}+\mathrm{O}\left(\mathrm{e}_{\mathrm{n}}^{3}\right)\right]$.

From (13) and (15), we have

$f^{\prime}\left(x_{n}\right)+f^{\prime}\left(x_{n+1}^{*}\right)=2 f^{\prime}(\alpha)\left[1+\mathrm{c}_{2} \mathrm{e}_{\mathrm{n}}+\left(\mathrm{c}_{2}^{2}+\frac{3}{2} \mathrm{c}_{3}\right) \mathrm{e}_{\mathrm{n}}^{2}+\mathrm{O}\left(\mathrm{e}_{\mathrm{n}}^{3}\right)\right]$,

$2 f^{\prime}\left(x_{n}\right) f^{\prime}\left(x_{n+1}^{*}\right)=2 f^{\prime}(\alpha)^{2}\left[1+2 c_{2} e_{n}+\left(2 c_{2}^{2}+3 c_{3}\right) \mathrm{e}_{n}^{2}+O\left(e_{n}^{3}\right)\right]$.

Dividing (17) by (16), we get

$\frac{2 f^{\prime}\left(x_{n}\right) f^{\prime}\left(x_{n+1}^{*}\right)}{f^{\prime}\left(x_{n}\right)+f^{\prime}\left(x_{n+1}^{*}\right)}=f^{\prime}(\alpha)\left[1+\mathrm{c}_{2} \mathrm{e}_{\mathrm{n}}+\frac{3}{2} \mathrm{c}_{3} \mathrm{e}_{\mathrm{n}}^{2}+\mathrm{O}\left(\mathrm{e}_{\mathrm{n}}^{3}\right)\right]$.

Further, we have

$\frac{\mathrm{x}_{\mathrm{n}+1}^{*}+\mathrm{x}_{\mathrm{n}}}{2}=\alpha+\frac{1}{2} \mathrm{e}_{\mathrm{n}}+\frac{1}{2} \mathrm{c}_{2} \mathrm{e}_{\mathrm{n}}^{2}+\left(\mathrm{c}_{3}-\mathrm{c}_{2}^{2}\right) \mathrm{e}_{\mathrm{n}}^{3}+\mathrm{O}\left(\mathrm{e}_{\mathrm{n}}^{4}\right)$.

Again expanding $f^{\prime}\left(\frac{x_{n+1}^{*}+x_{n}}{2}\right)$ by Tailor's series about $\alpha$ and using (19), we get

$2 f^{\prime}\left(\frac{\mathrm{x}_{\mathrm{n}+1}^{*}+\mathrm{x}_{\mathrm{n}}}{2}\right)=2 f^{\prime}(\alpha)\left[1+\mathrm{c}_{2} \mathrm{e}_{\mathrm{n}}+\left(\mathrm{c}_{2}^{2}+\frac{3}{4} \mathrm{c}_{3}\right) \mathrm{e}_{\mathrm{n}}^{2}+\mathrm{O}\left(\mathrm{e}_{\mathrm{n}}^{3}\right)\right]$.

Adding (18) and (20), we get

$\frac{2 f^{\prime}\left(x_{n}\right) f^{\prime}\left(x_{n+1}^{*}\right)}{f^{\prime}\left(x_{n}\right)+f^{\prime}\left(x_{n+1}^{*}\right)}+2 f^{\prime}\left(\frac{x_{n+1}^{*}+x_{n}}{2}\right)=3 f^{\prime}(\alpha)\left[1+\mathrm{c}_{2} \mathrm{e}_{\mathrm{n}}+\left(\frac{2}{3} \mathrm{c}_{2}^{2}+\mathrm{c}_{3}\right) \mathrm{e}_{\mathrm{n}}^{2}+\mathrm{O}\left(\mathrm{e}_{\mathrm{n}}^{3}\right)\right]$.

Then, from equations(12)and (21), we get

$\frac{3 f\left(x_{n}\right)}{\frac{2 f^{\prime}\left(x_{n}\right) f^{\prime}\left(x_{n+1}^{*}\right)}{f^{\prime}\left(x_{n}\right)+f^{\prime}\left(x_{n+1}^{*}\right)}+2 f^{\prime}\left(\frac{x_{n+1}^{*}+x_{n}}{2}\right)}=e_{n}-\frac{2}{3} c_{2}^{2} e_{n}^{3}+O\left(e_{n}^{4}\right)$.

The above equation (22) is substituted in (11)to get

$x_{n+1}=x_{n}-\left[e_{n}-\frac{2}{3} c_{2}^{2} e_{n}^{3}+O\left(e_{n}^{4}\right)\right]$.Since $x_{n}=e_{n}+\alpha$, we finally obtain that

$e_{n+1}=\frac{2}{3} c_{2}^{2} e_{n}^{3}+O\left(e_{n}^{4}\right)$. This shows that the method (11) has third order convergence.

\section{Numerical Examples}

In this section, we present the results of numerical calculations to compare the efficiency of HarmonicSimpson-Newton's method (HSN). We compare HSN with Newton's method (NM) and other cubic convergent 
methods given in section III. We note thatfunction $f_{5}(x)$ is not convergent inAN method if $x_{0}=-1$ even when 100 iterations are used, but convergent in the present method.Stopping criterion for the iterations has been taken as $\left|\mathrm{x}_{\mathrm{n}+1}-\mathrm{x}_{\mathrm{n}}\right|+\left|\mathrm{f}\left(\mathrm{x}_{\mathrm{n}+1}\right)\right|<\varepsilon$, where $\varepsilon=10^{-14}$.

Different functions and number of iterations to find their roots are given in Table1. Computational order of convergence (COC) and the number of function evaluation (NOFE) are given in Table 2. Numerical computations are performed in the Matlab software with double precision accuracy and the results are presented.

Table 1

\begin{tabular}{|c|c|c|c|c|c|c|c|c|}
\hline \multirow{2}{*}{$f(x)$} & \multirow{2}{*}{$x_{0}$} & \multicolumn{6}{|c|}{ Number of iterations } & \multirow[t]{2}{*}{ Root } \\
\hline & & NM & AN & $\mathrm{MN}$ & $\mathrm{TN}$ & SN & HSN & \\
\hline \multirow{2}{*}{$f_{1}(x)=x^{3}+x^{2}-2$} & 1.5 & 6 & 5 & 4 & 5 & 5 & 4 & \multirow[t]{2}{*}{$\alpha=1$} \\
\hline & 3 & 8 & 6 & 6 & 6 & 6 & 5 & \\
\hline \multirow{2}{*}{$f_{2}(x)=\operatorname{Sin}(x)+x \operatorname{Cos}(x)$} & 1.3 & 6 & 5 & 5 & 5 & 5 & 5 & \multirow[t]{2}{*}{$\alpha=2.028757838110434$} \\
\hline & 3 & 6 & 5 & 4 & 4 & 4 & 4 & \\
\hline \multirow{2}{*}{$f_{3}(x)=x^{2}-e^{x}-3 x+2$} & 3 & 7 & 5 & 5 & 5 & 5 & 5 & \multirow[t]{2}{*}{$\alpha=0.257530285439861$} \\
\hline & 5 & 9 & 7 & 6 & 6 & 6 & 6 & \\
\hline \multirow[t]{2}{*}{$f_{4}(x)=x \mathrm{e}^{\mathrm{x}}-1$} & 3 & 9 & 7 & 6 & 7 & 6 & 6 & \multirow[t]{2}{*}{$\alpha=0.567143290409784$} \\
\hline & 4.5 & 11 & 8 & 8 & 8 & 8 & 7 & \\
\hline \multirow[t]{2}{*}{$f_{5}(x)=x^{3}-10$} & 3 & 6 & 5 & 5 & 5 & 5 & 4 & \multirow[t]{2}{*}{$\alpha=2.154434690031884$} \\
\hline & -1 & 7 & $\mathrm{NC}$ & 6 & 11 & 7 & 5 & \\
\hline \multirow[t]{2}{*}{$f_{6}(x)=\mathrm{e}^{\mathrm{x}}+\mathrm{x}-20$} & 10 & 13 & 9 & 9 & 9 & 9 & 8 & \multirow[t]{2}{*}{$\alpha=2.842438953784447$} \\
\hline & 5 & 8 & 6 & 4 & 6 & 6 & 5 & \\
\hline
\end{tabular}

Table 2

\begin{tabular}{|c|c|c|c|c|c|c|c|c|c|c|c|c|c|}
\hline \multirow{2}{*}{$f(x)$} & \multirow{2}{*}{$x_{0}$} & \multicolumn{6}{|l|}{$\mathrm{COC}$} & \multicolumn{6}{|c|}{ NOFE } \\
\hline & & NM & AN & $\mathrm{MN}$ & TN & SN & HSN & NM & AN & $\mathrm{MN}$ & TN & SN & HSN \\
\hline \multirow[t]{2}{*}{$f_{1}(x)$} & 1.5 & 2.00 & 2.99 & 2.75 & 2.99 & 2.99 & 2.80 & 12 & 15 & 12 & 20 & 20 & 16 \\
\hline & 3 & 2.00 & 2.98 & 2.99 & 2.99 & 2.99 & 2.80 & 16 & 18 & 18 & 24 & 24 & 20 \\
\hline \multirow[t]{2}{*}{$f_{2}(x)$} & 1.3 & 1.99 & 3.82 & 3.17 & 3.07 & 3.12 & 3.08 & 12 & 15 & 15 & 20 & 20 & 20 \\
\hline & 3 & 1.99 & 4.10 & 2.71 & 2.88 & 2.60 & 2.70 & 12 & 15 & 12 & 16 & 16 & 16 \\
\hline \multirow[t]{2}{*}{$f_{3}(x)$} & 3 & 2.00 & 3.04 & 3.39 & 2.91 & 2.66 & 2.78 & 14 & 15 & 15 & 20 & 20 & 20 \\
\hline & 5 & 2.00 & 3.01 & 3.29 & 3.20 & 3.16 & 2.69 & 18 & 21 & 18 & 24 & 24 & 24 \\
\hline \multirow[t]{2}{*}{$f_{4}(x)$} & 3 & 2.00 & 2.99 & 2.88 & 2.99 & 2.84 & 2.93 & 18 & 21 & 18 & 28 & 24 & 24 \\
\hline & 4.5 & 2.00 & 2.96 & 2.99 & 2.98 & 2.99 & 2.91 & 22 & 24 & 24 & 32 & 32 & 28 \\
\hline \multirow[t]{2}{*}{$f_{5}(x)$} & 3 & 2.00 & 2.99 & 2.99 & 2.99 & 2.99 & 2.81 & 12 & 15 & 15 & 20 & 20 & 16 \\
\hline & -1 & 2.00 & NC & 2.91 & 3.01 & 3.02 & 2.95 & 14 & NC & 18 & 44 & 36 & 20 \\
\hline \multirow[t]{2}{*}{$f_{6}(x)$} & 10 & 2.00 & 2.93 & 3.00 & 2.98 & 2.99 & 2.94 & 26 & 27 & 27 & 36 & 28 & 32 \\
\hline & 5 & 2.00 & 2.99 & 3.00 & 2.99 & 2.99 & 2.94 & 16 & 18 & 12 & 24 & 24 & 20 \\
\hline
\end{tabular}

$\mathrm{NC}-$ Not Convergent, $\mathrm{X}_{0}$ - initial point

\section{Discussion}

The basic advantage of the present method is that it is a variant of Newton's method based on Newton's theorem. Comparing to other cubic convergent methods, our method converges faster and takes less number of iterations. Further,this method does not require the computation of second or higher order derivatives although it produces third order convergence. This method can easily be extended for equations having multiple roots and for multi-variable case.

\section{References}

[1] M.K. Jain, S.R.K. Iyengar, R.K. Jain, Numerical Methods for Scientific and Engineering Computation, New Age International, $6^{\text {th }}$ edition, 2012.

[2] S. Weerakoon. T.G.I. Fernando, A variant of Newton's method with accelerated third-order convergence, Applied Mathematics Letters $13(8)(2000)$ 87-93. 
[3] V. I. Hasanov, I. G. Ivanov, G. Nedjibov, A new modification of Newton's method, Applied Mathematics and Engineering 27 (2002) 278 -286.

[4] G. Nedzhibov, On a few iterative methods for solving nonlinear equations. Application of Mathematics in Engineering and Economics'28, in: Proceeding of the XXVIII Summer school Sozopol' 02, pp.1-8, Heron press, Sofia, 2002.

[5] M. Frontini, E. Sormoni, Some variants of Newton's method with third order convergence, Applied Mathematics and Computation 140 (2003) 419-426.

[6] A.Y. Ozban, Some new variants of Newton's method, Applied Mathematics Letters 17 (2004) 677-682.

[7] H.H.H. Homeier, On Newton type methods with cubic convergence, Journal of Computational and Applied Mathematics 176 (2005) 425-432.

[8] D.K.R. Babajee, M.Z. Dauhoo, An analysis of the properties of the variants of Newton's method with third order convergence, Applied Mathematics and Computation 183 (2006) 659-684.

[9] K. Jisheng, L.Yitian, W. Xiuhua, Third-order modification of Newton's method, Journal of Computational and Applied Mathematics 205 (2007) $1-5$.

[10] T. Lukic, N. M. Ralevic, Geometric mean Newton's method for simple and multiple roots, Applied Mathematics Letters 21 (2008) 30-36.

[11] O. Y. Ababneh, New Newton's method with third-order Convergence for solving nonlinear equations, World Academy of Science, Engineering and Technology 61 (2012) 1071-1073. 